Edición Extraordinaria. p.p. 311 - 318

Memorias del IX Encuentro Nacional de Experiencias en Enseñanza de la Biología y la Educación Ambiental. IV Congreso Nacional de Investigación en Enseñanza de la Biología.

\title{
EL CONOCIMIENTO PROFESIONAL DEL PROFESOR DE BIOLOGÍA EN RELACIÓN CON EL APRENDIZAJE DEL CONCEPTO ÁCIDO NUCLEICO
}

\section{THE PROFESSIONAL KNOWLEDGE OF THE TEACHER OF BIOLOGY ABOUT THE LEARNING OF THE NUCLEIC ACIDS CONCEPT}

\section{Andrea Serna Dimas ${ }^{1}$}

\section{RESUMEN}

El Conocimiento Profesional del Profesor surge como campo de investigación ante la creciente necesidad de la época por develar aquello que piensa el profesor respecto a las prácticas educativas y cómo estas son concebidas. En 1968, se celebraría el Congreso del National Institute of Education, en donde Lee Shulman en conjunto con un grupo de panelistas presentan una propuesta cuyo objetivo sería describir la vida mental de los profesores de tal forma que, al finalizar la presentación, los panelistas llegaron a concluir una concepción del profesor como un "agente que toma decisiones, reflexiona, emite juicios, tiene creencias, actitudes, entre otras actividades".

Este misma línea de investigación, Conocimiento Profesional del Profesor, plantea entonces una serie de aspectos involucrados en los procesos que adelanta el profesor en el aula, dentro de los cuales se incluyen sus concepciones respecto a la materia que enseña, el cómo la enseña y cuáles son sus propósitos, implícitos y explícitos, respecto al aprendizaje. Específicamente, en la enseñanza de las ciencias, se relaciona el modelo de formación al cual responde el profesor como un factor influyente en la manera como este presenta los contenidos de las ciencias, particularmente, de la biología.

De tal manera que, el determinar cómo el Conocimiento Profesional del Profesor puede influir en los procesos de enseñanza y aprendizaje que se dan en el aula respecto a un concepto específico, constituye un aporte a la construcción del marco teórico que enmarca este campo de investigación, así como la producción y ejecución de prácticas educativas reflexionadas.

\section{PALABRAS CLAVE}

Conocimiento Profesional del Profesor, Enseñanza-Aprendizaje, Ácido Nucleico, Constructivismo

\footnotetext{
${ }^{1}$ Universidad Distrital Francisco José de Caldas
} 
Bio - grafía. Escritos sobre la Biología y su Enseñanza. ISSN 2027-1034

Edición Extraordinaria. p.p. 311 - 318

Memorias del IX Encuentro Nacional de Experiencias en Enseñanza de la Biología y la Educación Ambiental. IV Congreso Nacional de Investigación en Enseñanza de la Biología.

\section{ABSTRACT}

The Professional Knowledge of the Teacher appeared as field of investigation for the current needs to discover the thought of the teacher within educational practices and how these are conceived. In 1968 in the Congress of the National Institute of Education a group of panelists headed by Lee Shulman presented a proposal which objective was to describe the mental life of the teachers. The panelists concluded that the teacher was an "agent who takes decisions, thinks, judgments, that has beliefs, attitudes, between other activities" (Perafán 2005, p. 15).

The investigation about the Professional Knowledge of the Teacher takes as question a series of processes within the practice of the teacher in the classroom, includes his conceptions about the object, how it teaches and which are his implicit and explicit intentions around the learning. Specifically, in the education of the sciences this approach indicates that the model of formation of the teacher is an influential factor in the way as he presents the contents of the sciences.

The clarification of the way like the Professional Knowledge of the Teacher can influence the processes of education - learning within classroom constitutes an contribution to the theoretical developments of this field of investigation as well as in the creation of educational reflexive practices.

\section{KEY WORDS}

Professional Knowledge of the Teacher, teaching - learning, Nucleic acids, Constructivism.

\section{INTRODUCCIÓN}

El trabajo aquí planteado, es un proyecto que se encuentra en un proceso de elaboración sustentado desde la investigación educativa EL CONOCIMIENTO PROFESIONAL DEL PROFESOR DE BIOLOGÍA EN RELACIÓN CON LOS PROCESOS DE ENSEÑANZA y APRENDIZAJE DEL CONCEPTO ÁCIDO NUCLEICO, adscrito a la Facultad de Ciencias y Educación de la Universidad Distrital Francisco José de Caldas, Bogotá D.C., Colombia.

Desde un ejercicio de corte cualitativo y un referente epistemológico ontológico de tipo interpretativo, se caracteriza el conocimiento profesional del profesor de biología y se analizan las posibles relaciones entre este conocimiento y la enseñanza y aprendizaje del concepto ácido nucleico.

La estructura del texto está determinada a partir de tres ejes temáticos; el primero es conocimiento profesional del profesor, donde se amplía elementos de orden teóricoconceptual, desde lo histórico y cómo se relaciona con la enseñanza-aprendizaje de las ciencias.

El segundo tema, sobre los procesos de enseñanza y aprendizaje de las ciencias, desde los modelos de formación y teorías, así como las concepciones de los profesores sobre su enseñanza, en términos de concepciones científicas y didácticas. 
Bio - grafía. Escritos sobre la Biología y su Enseñanza. ISSN 2027-1034

Edición Extraordinaria. p.p. 311 - 318

Memorias del IX Encuentro Nacional de Experiencias en Enseñanza de la Biología y

la Educación Ambiental. IV Congreso Nacional de Investigación en Enseñanza de la Biología.

El tercer aspecto a desarrollar, es el concepto de ácido nucleico a partir de una aproximación a un referente histórico y epistemológico de su concepción y evolución teórica.

Además, se presenta una caracterización inicial de los sujetos de estudio, atendiendo al estudio de casos como método seleccionado para la ejecución de la propuesta investigativa.

Los aportes de esta investigación educativa se enmarcan en la concepción de profesor que presenta el campo de investigación sobre Conocimiento Profesional del Profesor, los elementos que lo caracterizan y que determinan su práctica diaria en el aula y fuera de esta, así como las relaciones que se establecen durante la enseñanza de un concepto particular en la biología.

El ejercicio presentado tiene como fines, caracterizar el conocimiento profesional del profesor (CPP) y reconocer la posible relación entre CPP y la enseñanza y aprendizaje del concepto ácido nucleico, desde el punto de vista teórico para aproximarse a un proceso de caracterización del profesor en la práctica y de los procesos que se presenten en el aula con respecto a los estudiantes.

\section{El conocimiento profesional del profesor: investigaciones iniciales}

La investigación sobre el conocimiento profesional del profesor se ubica:

A partir de 1975, época desde la que se registra un paulatino aumento del interés de los investigadores en educación por describir y comprender las propias interpretaciones que del proceso educativo, en general, y de los procesos de enseñanza-aprendizaje en las aulas, en particular, mantienen los docentes. (Perafán, 2005, p. 15)

Así, a partir de 1975, en diferentes países, un buen número de académicos inician trabajos de investigación que buscan dar cuenta del pensamiento del profesor como una condición fundamental que explica la posibilidad del desarrollo docente, y que permite comprender las diferentes prácticas de enseñanza. (Perafán, 2005, p. 16)

Inicialmente, los aportes a la investigación se limitan al Pensamiento del Profesor como producto de los aportes que realizaría la psicología cognitiva. Sin embargo, y gracias a los aportes de la antropología, la etnografía y la sociología, el campo de investigación fue ampliado hacia el qué y cómo conocen los profesores, de tal manera que cambia de nombre al que conocemos en la actualidad como conocimiento de los profesores.

Esta nueva concepción se explica:

Como un cambio de interés motivado por el descubrimiento de la estrechez del análisis psicológico y cognitivo de las investigaciones, así como de una creciente influencia contextual y epistemológica que reconocía, el hecho evidente de que los docentes son profesionales que desarrollan una epistemología de la práctica, es decir, que generan conocimiento sobre la enseñanza que merece la pena ser investigado. (Marcelo, 2005, p. 47) 
Bio - grafía. Escritos sobre la Biología y su Enseñanza. ISSN 2027-1034

Edición Extraordinaria. p.p. 311 - 318

Memorias del IX Encuentro Nacional de Experiencias en Enseñanza de la Biología y la Educación Ambiental. IV Congreso Nacional de Investigación en Enseñanza de la Biología.

De allí en adelante, surgirían numerosas investigaciones cuyo objeto de estudio sería el profesor y su conocimiento. Siendo las décadas de los ochenta y noventa, como las que mayor aporte teórico realizarín al campo así como evolución en su construcción y planteamientos.

Sobre este aporte teórico, cabe resaltar las aportaciones hechas por Shulman (1986), así como los de Malinowski (1995), Schon (1992-1998), Dewey (1989) y Dennet (1996-1998) acerca del profesor como profesional o como práctico reflexivo. (Perafán, 2005, p. 15)

Así mismo, el conocimiento de los profesores también ha influido en investigaciones sobre la didáctica, de tal manera que se considera en la actualidad como una línea prioritaria de investigación (Porlán \& Rivero, 1998). Al respecto, señalan los mismos autores que "los profesores son los únicos que pueden hacer evolucionar el modelo de enseñanza predominante...conviene investigar sus concepciones científicas, didácticas y curriculares". (p.98)

EI CPP en la enseñanza y aprendizaje de las ciencias

La investigación sobre el conocimiento profesional del profesor (CPP) en relación con los procesos de enseñanza y aprendizaje de las ciencias, se fundamenta en la investigación de las didácticas específicas, cuyos trabajos se refieren hacia los finales de los ochenta (Porlán \& Rivero, 1998, p. 97).

Es entonces que, una vez establecida la figura del profesor como sujeto que produce un conocimiento como resultado de su práctica diaria, surge la necesidad de establecer cómo ese conocimiento influencia de alguna manera las decisiones que este toma en el ejercicio de su práctica y cómo, a su vez, estas decisiones afectan o determinan el aprendizaje de las ciencias.

Algunos autores han puesto en evidencia la naturaleza compleja de la enseñanza; complejidad debida a diferentes factores, desde los sociales y culturales hasta los relacionados con el conocimiento y creencias, tanto de los profesores como de los estudiantes. (Moreno, 2005, p. 64)

Autores como Porlán \& Rivero (1998), manifiestan la influencia de los modelos de formación del profesorado en la manera cómo estos realizan la enseñanza de la ciencia durante el ejercicio de su profesión. A través de las investigaciones de Pérez Gómez (1992), se sugiere la necesidad de analizar las diferentes perspectivas existentes en la formación del profesorado de tal manera que se puedan relacionar con la función que asume el docente y el modelo de enseñanza que persigue. (Porlán \& Rivero, 1998, p. 14)

\section{El concepto de ácido nucleico}

Aunque si bien el conocimiento científico común ha atribuido el descubrimiento de la molécula de $A D N$ a los científicos Watson y Crick, fueron muchos otros quienes sentaron las bases de la estructura propuesta por estos científicos y a quienes se les ha restado importancia en los aportes que realizaron en el estudio sobre los ácidos nucleicos.

Sobre este hecho, escribe (Olivera, 2003) "al igual que la memoria, la historia de la ciencia tiende a simplificar los hechos, a veces hasta el punto de caricaturizarlos". ( $p$. 
Bio - grafía. Escritos sobre la Biología y su Enseñanza. ISSN 2027-1034

Edición Extraordinaria. p.p. 311 - 318

Memorias del IX Encuentro Nacional de Experiencias en Enseñanza de la Biología y la Educación Ambiental. IV Congreso Nacional de Investigación en Enseñanza de la Biología.

5) En consecuencia, el origen de las investigaciones sobre los ácidos nucleicos se remite a las investigaciones sobre el ADN de tal forma que, la literatura referencia principalmente los trabajos adelantados en torno a la estructura de esta molécula.

Sin embargo, en investigaciones relacionadas, se sugiere el origen de su concepto desde los antiguos griegos sobre la pangénesis y la presencia de ciertas secreciones del cuerpo de los padres que se mezclaban para dar origen a un hijo. (Olivera, 2003, p. 6)

Señala el mismo Olivera (2003) que, sería entonces el siglo XIX el que arrojaría otras interpretaciones respecto a la herencia de los caracteres y su origen, relacionando los trabajos del bioquímico suizo Friedrich Miescher, quien en 1869 e interesado por estudiar la composición química de las células, descubriría en células linfoides la presencia de una extraña sustancia que luego de aislar del núcleo, denominaría entonces como nucleína. Pero por diversos acontecimientos de la época los hallazgos de Miescher no fueron publicados sino hasta 1871. (Olivera, 2003, p.7)

A partir de los trabajos de Miescher muchos otros científicos se verían atraídos hacia el estudio de la nucleína, siendo Robert Altmann en 1889 quien emplearía el término de ácido nucleico para referirse a las proteínas presentes en el núcleo de la célula pero que contenían un carácter ácido.

\section{METODOLOGÍA}

Los presupuestos metódicos y metodológicos de la investigación propuesta, suponen la apropiación de las características atribuidas al investigador cualitativo, siendo este reflexivo, analítico, multimetódico y cuyo trabajo se centra en estudiar las personas, las historias, los comportamientos; así mismo, el investigador de orden cualitativo hace uso de un lenguaje corriente y de tipo narrativo para presentar el o los hechos a estudiar, y siendo fundamentalmente la comunicación quien medie los procesos que se adelanten durante la ejecución del proceso investigativo (Arnal, Rincón, \& Latorre, 1992., Denzin \& Lincoln, 1994., Stake, 1999., Vasilachis, 2006).

Así mismo, durante la selección del estudio de caso es conveniente atender a los criterios propuestos por Stake (1999) respecto a "... rentabilidad de aquello que se aprende a través del caso seleccionado; la facilidad para ser abordado; y los resultados de una valoración preliminar". (p. 15)

Por lo tanto, se espera que la selección del estudio de casos, como metodología a seguir para el proceso investigativo permita la consecución de los objetivos propuestos y sea un mecanismo útil en el proceso interpretativo de los datos recogidos, permitiendo un análisis riguroso y el debate respecto a aquellas generalizaciones que permiten la comprensión del caso en particular.

A continuación, se relaciona el proceso metodológico de la propuesta investigativa, el cual se encuentra establecido en cinco fases de desarrollo.

Tabla 1

Fases de desarrollo de la propuesta de investigación El conocimiento profesional del profesor de biología en relación con los procesos de enseñanza y aprendizaje del concepto ácido nucleico 
Bio - grafía. Escritos sobre la Biología y su Enseñanza. ISSN 2027-1034

Edición Extraordinaria. p.p. 311 - 318

Memorias del IX Encuentro Nacional de Experiencias en Enseñanza de la Biología y la Educación Ambiental. IV Congreso Nacional de Investigación en Enseñanza de la Biología.

\begin{tabular}{|c|c|c|}
\hline Fase & Descripción & Producto \\
\hline $\begin{array}{lrr}\text { Fase } & \text { I. } & \text { Revisión } \\
\text { bibliográfica } & \text { y } \\
\text { construcción } & \text { del } & \text { marco } \\
\text { teórico } & & \end{array}$ & $\begin{array}{l}\text { Fase en ejecución, que } \\
\text { pretende construir la } \\
\text { estructura conceptual y } \\
\text { metodológica de la } \\
\text { investigación. }\end{array}$ & $\begin{array}{l}\text { Anteproyecto } \\
\text { investigación } \\
\text { Marco conceptual } \\
\text { Diseño metodológico } \\
\text { Estudio de casos }\end{array}$ \\
\hline $\begin{array}{lcr}\text { Fase II. } & \text { Diseño } & \text { y } \\
\text { preparación } & \text { de } & \text { los } \\
\text { instrumentos } & & \end{array}$ & $\begin{array}{l}\text { Fase inicial para el trabajo } \\
\text { de campo que implica la } \\
\text { revisión bibliográfica } \\
\text { acerca del estudio de caso } \\
\text { y la elaboración de los } \\
\text { instrumentos requeridos } \\
\text { para el desarrollo de la } \\
\text { investigación. También } \\
\text { requiere la presentación } \\
\text { en el lugar donde se } \\
\text { desarrollara la la } \\
\text { investigación y la la } \\
\text { entrevista inicial con los } \\
\text { sujetos participantes. }\end{array}$ & $\begin{array}{l}\text { Instrumentos de } \\
\text { investigación: elaboración } \\
\text { de los cuestionarios para } \\
\text { las entrevistas } \\
\text { semiestructuradas; } \\
\text { desarrollo de formatos de } \\
\text { observación de clases; etc. }\end{array}$ \\
\hline $\begin{array}{l}\text { Fase III. Ejecución del } \\
\text { trabajo de campo }\end{array}$ & $\begin{array}{l}\text { Observación de los } \\
\text { sujetos y aplicación de los } \\
\text { instrumentos; descripción } \\
\text { de contextos, entrevistas. }\end{array}$ & Recolección de datos \\
\hline $\begin{array}{l}\text { Fase IV. Análisis e } \\
\text { interpretación }\end{array}$ & $\begin{array}{l}\text { Organización de los datos } \\
\text { recolectados } \\
\text { interpretación según el } \\
\text { modelo de CPP elegido. }\end{array}$ & $\begin{array}{l}\text { Triangulación y validación } \\
\text { de los casos estudiados }\end{array}$ \\
\hline $\begin{array}{l}\text { Fase V. Caracterización } \\
\text { del CPP de biología y } \\
\text { construcción del informe }\end{array}$ & $\begin{array}{l}\text { Elaboración del informe } \\
\text { final a partir del trabajo } \\
\text { desarrollado. }\end{array}$ & $\begin{array}{l}\text { Caracterización del CPP de } \\
\text { biología } \\
\text { Análisis de las } \\
\text { observaciones realizadas in } \\
\text { situ con el fin de hallar } \\
\text { posibles relaciones entre el } \\
\text { CPP y el aprendizaje de los } \\
\text { estudiantes } \\
\text { Informe final } \\
\text { Artículo de aporte a la línea } \\
\text { de investigación }\end{array}$ \\
\hline
\end{tabular}

Descripción de las fases de desarrollo de la propuesta de investigación y los productos esperados al final de cada fase. 
Bio - grafía. Escritos sobre la Biología y su Enseñanza. ISSN 2027-1034

Edición Extraordinaria. p.p. 311 - 318

Memorias del IX Encuentro Nacional de Experiencias en Enseñanza de la Biología y la Educación Ambiental. IV Congreso Nacional de Investigación en Enseñanza de la Biología.

\section{Caracterización inicial}

La propuesta de investigación pretende realizarse en un colegio privado del norte de la ciudad. Este colegio es de tipo femenino y se caracteriza por el empleo del sistema preventivo de Don Bosco como elemento central de su filosofía institucional.

El caso "Ofelia" se estudiará a partir de una docente licenciada en química de la Universidad Distrital Francisco José de Caldas, con cerca de 20 años de experiencia en colegios públicos y privados de la ciudad. Durante esta caracterización inicial, se concibe así misma como "una profesora con capacidad para fomentar en los estudiantes la adquisición de valores frente a la naturaleza y a la sociedad; as ícomo el desarrollo de habilidades para relacionar la ciencia con la vida social y su uso en la solución de problemas. En términos generales, se manifiesta disponible al cambio y a las innovaciones para beneficio de la comunidad educativa".

El grupo de estudiantes con el que se trabajará para ejecutar la parte de la propuesta que busca establecer las posibles relaciones entre el CPP y el aprendizaje, corresponde con un grupo de 16 niñas entre los 14 y 16 años de edad; de estrato socieconómico 3, en su mayoría de padres profesionales y con trabajos estables.

\section{CONCLUSIONES}

El conocimiento profesional del profesor se presenta como un concepto desconocido para la mayoría de profesionales que trabajan en el campo de la docencia y desconocen a profundidad las implicaciones de su práctica. Este conocimiento que se establece hace ya algunos años como campo de investigación, resulta ser de carácter relevante para la construcción de marcos teóricos que ayuden a la fundamentación de la profesionalización de la licenciatura y se presenta como una oportunidad de reflexión en torno a la educación como práctica reflexionada.

Los aportes teóricos provenientes del país de origen de esta línea de investigación, sumados a otros países que se interesaron en este tema, constituyeron para nuestro país, Colombia, una base de reflexión y análisis de las prácticas educativas y han permitido el replanteamiento y hallazgo de importantes observaciones que enriquecen la construcción de esos marcos teóricos.

Se espera que ejercicios de caracterización del CPP como el propuesto en este artículo, aporte no sólo a la línea de investigación constituída en nuestro país, sino también, permita avanzar hacia nuevas formas de pensar la docencia como una profesión provista de un conocimiento propio que se desarrolla en y para la práctica y que merece ser descrito.

Reconocer cuáles son las relaciones entre ese CPP y el aprendizaje de los estudiantes se observa como una necesidad del campo de investigación que aún no se ha clarificado pero que podría aportar a la didáctica de las ciencias y a la mejora de las prácticas educativas.

El concepto de ácido nucleico se encuentra provisto de un interés particular en la actualidad, que implica múltiples interpretaciones y requiere de una clara presentación en el aula por parte del docente que lo propone. Al parecer, la forma en que este se 
Bio - grafía. Escritos sobre la Biología y su Enseñanza. ISSN 2027-1034

Edición Extraordinaria. p.p. 311 - 318

Memorias del IX Encuentro Nacional de Experiencias en Enseñanza de la Biología y la Educación Ambiental. IV Congreso Nacional de Investigación en Enseñanza de la Biología.

enseña y aprende tiene alguna relación con el concepto de ciencia que desarrolla el profesor durante sus procesos formativos y con el que manifiesta en el aula.

\section{BIBLIOGRAFÍA}

Marcelo, C. (2005). La investigación sobre el conocimiento de los profesores y el proceso de aprender a enseñar. En G. Perafán, \& A. Aduriz-Bravo, Pensamiento y conocimiento de los profesores. Debate y perspectivas internacionales (págs. 47-61). Bogotá: Nomos.

Moreno, M. (2005). El pensamiento del profesor. Evolución y estado actual de las investigaciones. En G. Perafán, \& A. Aduríz-Bravo, Pensamiento y conocimiento de los profesores. Debate y perspectivas internacionales (págs. 63-79). Bogotá: Nomos.

Olivera, M. B. (2003). La construcción de la doble hélice. De la nucleína al ADN. Ciencias, 4-15.

Perafán, G. (2005). La investigación acerca de los procesos de pensamiento de los docentes. En G. Perafán, \& A. Aduriz-Bravo, Pensamiento y conocimiento de los profesores. Debates y perspectivas internacionales (págs. 15-31). Bogotá: Nomos.

Porlán, R., \& Rivero, A. (1998). El conocimiento de los profesores. Una propuesta formativa en el área de ciencias. Sevilla: Díada. 